Harold \& DeGarmo also question whether there was indeed a difference in the standard control condition (usual care) for participants in the US and UK studies. There are certainly likely to be differences in the nature and uses of group care between the two countries, given the differences in their child-welfare and juvenile-justice systems. However, the point we were making is that, in the USA, the MTFC programme for adolescents has been principally found to be successful when targeted at young offenders, in studies that have used a variety of measures of recorded reoffending to assess its effectiveness. ${ }^{1-3}$ This emphasis on the effectiveness of MTFC-A with young offenders is also clear from the programme developers' own website (www.mtfc.com). By contrast, the participants in our study were young people with complex emotional and behavioural difficulties, 93\% of whom were in care because of abuse or neglect and less than a third of whom had a recent criminal conviction. The differences between the populations served by MTFC-A are clearly evident in an article comparing outcomes for high-risk adolescent girls written by the programme developers in the USA and their English colleagues ${ }^{4}$ and may perhaps partly explain why the results of the English evaluation were less positive than those in the USA.

1 Chamberlain P. Comparative evaluation of foster care for seriously delinquent youth: a first step. Community Altern 1990; 2: 21-36.

2 Chamberlain P, Reid JB. Comparison of two community alternatives to incarceration for chronic juvenile offenders. J Consult Clin Psychol 1998; 66 624-33

3 Leve L, Chamberlain P, Reid JB. Intervention outcomes for girls referred from juvenile justice: effects on delinquency. J Consult Clin Psychol 2005; 75: 1181-4.

4 Rhoades K, Chamberlain P, Roberts R, Leve L. MTFC for high risk adolescent girls: a comparison of outcomes in England and the United States. J Child Adolesc Subst Abuse 2013; 22: 435-49.

Jonathan Green, Professor of Child and Adolescent Psychiatry, University of Manchester and Manchester Academic Health Sciences Centre. Email: jonathan.green@manchester.ac.uk; N. Biehal, Department of Social Policy and Social Work, University of York, York; C. Roberts, Centre for Biostatistics, Institute of Population Health, University of Manchester, Manchester; J. Dixon, Social Policy Research Unit (SPRU), University of York, York; C. Kay, Institute of Brain Behaviour and Mental Health, University of Manchester, Manchester; E. Parry, Mood Disorders Centre, University of Exeter, Exeter; J. Rothwell, A. Roby, D. Kapadia, Institute of Brain Behaviour and Mental Health, University of Manchester, Manchester; S. Scott Institute of Psychiatry, King's College London, London; I. Sinclair, Social Policy Research Unit (SPRU), University of York, York, UK.

doi: 10.1192/bjp.205.6.498b

\section{Are we reinforcing the anti-medical model?}

The results of Penttilä et al's meta-analysis emphasised the importance of the duration of untreated psychosis (DUP) in long-term recovery from schizophreniform illness. ${ }^{1}$ Timely initiation of effective treatment has been demonstrated to improve outcome, but the modality of treatment is currently under much debate. Robust evidence exists for the efficacy of antipsychotic medication $^{2}$ but recent studies have proposed psychological interventions, specifically cognitive-behavioural therapy (CBT), as an alternative first-line treatment.

In a recent randomised controlled trial, CBT was used as a single intervention, instead of conventional antipsychotic treatment. ${ }^{3}$ To our complete surprise, one of the exclusion criteria was treatment with antipsychotic drugs. We wonder how ethical approval was granted, despite Tiihonen et al's robust demonstration of reduced mortality over a considerable follow-up period for patients receiving antipsychotic medication. ${ }^{4}$ We feel that this will set a dangerous precedent of offering psychological treatment as an alternative to evidence-based treatment. In a clinical setting, adherence to drug treatment is already a significant issue and there is potential to reinforce the idea that antipsychotic medication is harmful and unnecessary. We feel that this would further disadvantage an already vulnerable group of patients.

This issue has recently received a fair degree of coverage in the media, with articles such as Freeman \& Freeman's piece in The Guardian fuelling long-held popular beliefs that antipsychotics are ineffective and in fact damaging to health. ${ }^{5}$ Given the well-documented drawbacks of antipsychotic drugs, it is understandable that patients and professionals will invest hope in non-drug alternatives. However, a large meta-analysis with over 3000 participants shows at best a small effect size for CBT. ${ }^{6}$ In reference to Penttilä et al's paper, we would be interested to read subgroup analyses of specific first-line treatments and wonder if outcomes would differ between modalities.

While we would endorse any treatment, drug or non-drug based, that is proven to reduce DUP, it is vital that we do not lose sight of the fact that antipsychotics are the only evidence-based first-line therapy in psychotic illness.

1 Penttilä $M$, Jääskeläinen $E$, Hirvonen $N$, Isohanni $M$, Miettunen J. Duration of untreated psychosis as predictor of long-term outcome in schizophrenia: systematic review and meta-analysis. Br J Psychiatry 2014; 205: 88-94.

2 Leucht S, Arbter D, Engel RR, Kissling W, Davis JM. How effective are secondgeneration antipsychotic drugs? A metaanalysis of placebo-controlled trials. Mol Psychiatry 2009; 14: 429-47.

3 Morrison AP, Hutton P, Wardle M, Spencer H, Barratt S, Brabban A, et al. Cognitive therapy for people with a schizophrenia spectrum diagnosis not taking antipsychotic medication: an exploratory trial. Psychol Med 2012; 42: 1049-56.

4 Tiihonen J, Lannqvist J, Wahlbeck K, Klaukka T, Niskanen L, Tanskanen A et al. 11-year follow-up of mortality in patients with schizophrenia: a population-based cohort study (FIN11 study). Lancet 2009; 374: 620-7.

5 Freeman D, Freeman J. At last, a promising alternative to antipsychotics for schizophrenia. The Guardian, 7 March 2014.

6 Jauhar S, McKenna PJ, Radua J, Fung E, Salvador R, Laws KR. Cognitivebehavioural therapy for the symptoms of schizophrenia: systematic review and meta-analysis with examination of potential bias. Br J Psychiatry 2014; 204: 20-9.

Dorothea C Bindman, Core Trainee, London Deanery, email: dorothea.bindman@ nhs.net; Mukesh Kripalani, Tees, Esk and Wear Valleys NHS Foundation Trust, UK

doi: 10.1192/bjp.205.6.499

Author's reply: Dr Bindman and Dr Kripalani have suggested an analysis of the association between DUP and outcomes in subgroups by specific first-line treatment modalities. Unfortunately, it was not possible to analyse this in our meta-analysis, since none of the original studies had used only one treatment modality, but a combination of them in the early phases of treatment. As Bindman \& Kripalani point out, and based on current knowledge of the efficacy of treatments in the early phase of schizophrenia, it would not be ethical to study treatment without antipsychotic medication in a first-episode clinical sample. ${ }^{1}$ Also, DUP is usually defined as ending at the initiation of antipsychotic medication, which in clinical practice usually occurs about the same time as other treatment modalities begin; therefore, the included studies give only a little information on the effects of different treatments. However, it is interesting to note that de Haan et al investigated the effect of delay in intensive psychosocial treatment by comparing this effect with delay in treatment with antipsychotic medication; and found that delay in psychosocial treatment may be a more important predictor of negative symptoms than delay in antipsychotic treatment.

The discussion about the possible effects of antipsychotics has been rather intense recently. However, the current guidelines for treatment of psychosis and schizophrenia clearly indicate that 
antipsychotic medications are effective and recommended treatment for active psychotic symptoms, ${ }^{1}$ though there is not so much evidence for the long term (i.e. several years of antipsychotic treatments ${ }^{3}$ ). Additionally, the clinical use of these medications is not always straightforward because of their known side-effects and the fact that, in all psychiatric disorders and other illnesses in medicine, there are always patients who do not want to take the recommended treatment. This seems to have been the case in the trial pointed out by Bindman \& Kripalani. ${ }^{4}$ When considering the long-term effects of antipsychotics, it is evident that the long-term treatment of psychosis needs to be developed further.

We agree that it would be dangerous to see different treatments as alternatives to each other, and it has been shown that in psychiatry a combination of different treatments is, in general, more effective than any of them alone. ${ }^{5}$ Psychotherapy in the early phase of illness could be effective not only in preventing psychosis at prodromal phase, but also in enhancing adherence to antipsychotic medication. ${ }^{1}$ Current treatment guidelines do not suggest that treatment of first-episode psychosis should include only antipsychotic medication without psychosocial treatment, but rather state that medication is one of the cornerstones of psychosis treatment. We believe there is still a lot to do in developing both medication and psychosocial treatments for schizophrenia, and hopefully active research can support this development.

1 National Institute for Health and Care Excellence. Psychosis and Schizophrenia in Adults: Treatment and Management (Clinical Guideline 178). NICE, 2014.

2 de Haan L, Linszen DH, Lenior ME, de Win ED, Gorsira R. Duration of untreated psychosis and outcome of schizophrenia: delay in intensive psychosocial treatment versus delay in treatment with antipsychotic medication. Schizophr Bull 2003; 29: 341-8.

3 Harrow M, Jobe TH, Faull RN. Does treatment of schizophrenia with antipsychotic medications eliminate or reduce psychosis? A 20-year multi-follow-up study. Psychol Med 2014; 44: 3007-16.

4 Morrison AP, Turkington D, Pyle M, Spencer H, Brabban A, Dunn G, et al. Cognitive therapy for people with schizophrenia spectrum disorders not taking antipsychotic drugs: a single-blind randomised controlled trial. Lancet 2014; 383: 1395-403.

5 Cuijpers P, van Straten A, Warmerdam L, Andersson G. Psychotherapy versus the combination of psychotherapy and pharmacotherapy in the treatment of depression: a meta-analysis. Depress Anxiety 2009; 26: 279-88.

Matti Penttilä, Oulu University Institute of Clinical Medicine and Oulu University Hospital, email: Matti.Penttila@oulu.fi; Erika Jääskeläinen, Noora Hirvonen, Matti Isohanni, Oulu University Hospital, Jouko Miettunen, Oulu University Hospital and Medical Research Center Oulu, Finland.

doi: 10.1192/bjp.205.6.499a

\section{Electronic monitoring of forensic patients}

Tully et al raise important questions about the introduction of electronic monitoring of forensic patients. ${ }^{1}$ Incidents of absconding by forensic patients can give rise to calls for increased security and surveillance. As the authors point out, adoption of electronic monitoring as a panacea for these problems is shortsighted. Tully et al cover many of the concerns about electronic monitoring but one area is missing: that the evidence we have from electronic monitoring in the criminal justice sector is primarily of its effects on recidivism and absence without leave during use; evidence is very limited on the effects after its use.

In other words, electronic monitoring must eventually cease. Is the use of electronic monitoring during community reintegration actually preparing the patient for greater freedom and their rehabilitation, or simply delaying reoffending? Criminal justice experience with electronic monitoring focuses almost entirely on its effectiveness during use, such as on bail or as an alternative to incarceration, usually combined with home detention. Electronic monitoring combined with home detention is superior to imprisonment in these studies, but we already know that noncustodial responses to crime in general have superior outcomes to incarceration (see, for example, Wermink et $a l^{2}$ ).

We know very little about outcomes after the use of electronic monitoring. Although the use of global positioning satellite (GPS) technology might improve the person's performance in following rules, it is not clear that this sort of rule following encourages the person in the ultimate tasks of forensic rehabilitation. Does it improve the therapeutic alliance to help the person make the life changes necessary to recover from illness and illness-related offending? Or does electronic monitoring seem a physical manifestation of distrust and create distance between the patient and the treatment team? If the only way that a person can safely have community contact is to wear an ankle bracelet, isn't it questionable whether they are ready for that level of community contact? Electronic monitoring may allow the person more apparent personal freedom than their clinical risk would otherwise allow. As Tully et al point out, adoption of the GPS technology may seem appealing, but its costs and effects are not clear and neither is its impact on therapeutic and community engagement. Short-term reductions in absence without leave might give the appearance of progress that the patient has not actually achieved. Long-term outcome is equally as important as short-term adherence.

1 Tully J, Hearn D, Fahy T. Can electronic monitoring (GPS 'tracking') enhance risk management in psychiatry? Br J Psychiatry 2014; 205: 83-5.

2 Wermink H, Blokland A, Nieuwbeerta P, Nagin D, Tollenaar N. Comparing the effects of community service and short-term imprisonment on recidivism: a matched samples approach. J Exp Criminol 2010; 6: 325-49.

Alexander I. F. Simpson, Chief of Forensic Psychiatry, Stephanie R Penney, Centre for Addiction and Mental Health, email: sandy.simpson@camh.ca

doi: 10.1192/bjp.205.6.500

Tully and colleagues ${ }^{1}$ justify the introduction of electronic monitoring of medium secure patients without indication of the size of the problem of absconding or the incidence of serious harm other than to reference an article in The Sun newspaper, which is neither informative nor free of bias.

Decisions around leave for patients detained within a medium secure unit are clearly complex. Consideration should always be given to the risk of absconding and associated risks if the patient were to abscond. Thus, patients who are at high risk of absconding and a serious risk to the public would not receive leave, whether they were tagged or not. Another factor is the clinical team's trust in that patient to use leave appropriately. Tagging patients would be a very clear indicator of a lack of such trust.

The suggestion that patients enter into electronic monitoring with consent is questionable: many patients in our experience abide by suggestions of their clinical team in order to progress through the system. Given that there is yet to be a strong argument that tagging is necessary and primarily in the patient's best interest (as opposed to a matter of public protection), can one justify this coercion? We would be very interested to know the process in which patients' perspectives were taken into account and whether this has altered the intervention.

Electronic monitoring would inform the clinical team if the patient were to breach the conditions of their leave in terms of approximate location and time of leave; however, it would not inform the team as to what that patient was doing with their leave and would not necessarily prevent serious incidents occurring, as 